UDK 528.711

\title{
A CNN BASED HYBRID APPROACH TOWARDS AUTOMATIC IMAGE REGISTRATION
}

\author{
Pattathal Vijayakumar Arun \\ College of Science \& Technology, Phuentsholing, Bhutan \\ E-mail: arunpv2601@gmail.com
}

Received 03 May 2013; accepted 18 September 2013

\begin{abstract}
Image registration is a key component of spatial analyses that involve different data sets of the same area. Automatic approaches in this domain have witnessed the application of several intelligent methodologies over the past decade; however accuracy of these approaches have been limited due to the inability to properly model shape as well as contextual information. In this paper, we investigate the possibility of an evolutionary computing based framework towards automatic image registration. Cellular Neural Network has been found to be effective in improving feature matching as well as resampling stages of registration, and complexity of the approach has been considerably reduced using corset optimization. CNN-prolog based approach has been adopted to dynamically use spectral and spatial information for representing contextual knowledge. The salient features of this work are feature point optimisation, adaptive resampling and intelligent object modelling. Investigations over various satellite images revealed that considerable success has been achieved with the procedure. Methodology also illustrated to be effective in providing intelligent interpretation and adaptive resampling.
\end{abstract}

Keywords: Cellular Neural Network, image analysis, registration, resampling, remote sensing.

Reference to this paper should be made as follows: Arun, P. V. 2013. A CNN based hybrid approach towards automatic image registration, Geodesy and Cartography 39(3): 121-128.

\section{Introduction}

Image registration deals with the determination of local similarity between images, and involves the calculation of spatial geometric transforms that aligns related images to a common observational framework. Registration is a critical component of various spatial analyses; however its accuracy is affected by various factors such as geometrical complexity, noise, vague boundaries, mixed pixel problems, and fine characteristics of detailed structures (Fonseca, Costa 2004). Automatic image registration approaches have been broadly categorized as area based and feature based, among which the former adopts a region specific strategy whereas the latter a feature based one. Increasing resolution of satellite images have limited the accuracy of area based strategies and in turn popularized object based approaches. Different existing feature based algorithms lack contextual interpretation capability and adopt computationally complex methods (Zitová, Flusser 2003). Efficiency of these methods are situation and image-specific due to involvement of various parameters like spatial and spectral resolution, sensor characteristics etc. (Viola, Wells 1997; Mohanalin et al. 2009).

Literature reveals a great deal of recent approaches towards feature based image registration techniques; however significant improvement in accuracy is not visible over the past decade (Wyawahare 2009; Zitová, Flusser 2003; Malviya, Bhirud 2009; Gouveia et al. 2012; Liu, Wu 2012). Line, point and curve based approaches found in literatures (Zitová, Flusser 2003; Jian, Vemuri 2005) can only compensate to simple differences between images and make use of one only kind of feature information. Soft computing techniques such as neural networks, genetic algorithms, and fuzzy logic followed by probabilistic concepts such as random field variations, have been extensively applied in this context (Liu, Wu 2012; Gouveia et al. 2012; Jack, Roux 1995; Chow et al. 2004; Jankó et al. 2006). Literature has also revealed many mutual information 
as well as intensity-based approaches (Klein et al. 2007; Viola, Wells 1997; Cvejic et al. 2006; Mohanalin et al. 2009). N-dimensional classifiers as well as random field concepts and different transformation techniques (SIFT, Wavelet etc.) have also been applied for accurate registration (Malviya, Bhirud 2009; Hong, Zhang 2008). Contextual information is essential for avoiding registration ambiguity and predicate calculus has been used for the purpose (Porway et al. 2008). Most of these approaches adopt common image interpretation keys namely tone, texture, pattern, colour etc. for feature matching; however consideration of shape is usually neglected (Wyawahare 2009). Limited accuracy of these methodologies may also be attributed to the dependency on traditional static resampling techniques (Liu, Wu 2012; Gouveia et al. 2012). Contextual information required for registration is usually represented using predicate calculus rule sets. However calculus approach fails to represent spatial relations effectively as it lack an image compatible form (Mitchell et al. 1996; Zitová, Flusser 2003).

We have investigated the possibility of using random modeling techniques for improving automatic registration approaches. Our studies have found that techniques such as Cellular Automata (CA) (Mitchell et al. 1996), Cellular Neural Network (Orovas, Austin 1997) and Multiple Attractor Cellular Automata (Sikdar et al. 2000) along with Genetic Algorithm (GA) (Jian, Vemuri 2005) can be efficiently used for modeling feature shapes. This approach has been adopted for improving the feature matching as well as resampling stages to achieve better registration accuracy. The Cellular Automata rules have been adopted for effective context representation as it can represent image rules more effectively (Orovas, Austin 1997). Spectral and spatial information have been combined to increase the object class seperability to yield higher registration accuracy (Mercier, Lennon 2003). An adaptive kernel strategy (Srivastava 2004) along with Support Vector Random Field (SVRF) (Schnitzspan et al. 2008; Lee et al. 2005; Hosseini, Homayouni 2009) has been adopted for achieving a semi-supervised classification approach. Main obstruct in the modelling of features using Cellular Neural Network (CNN) (Mitchell et al. 1996) approach was increased computational complexity, which has been effectively tackled using coreset (Agarwal et al. 2001) based approximation. Scale Invariant $\mathrm{Fe}$ ature Transforms (SIFT) (Lowe 2004) are invariant to image scaling and rotation, and partially invariant to change in illumination, and are extensively used for registration. We have used the object specific information for optimizing and refining SIFT feature point descriptors.

Most of the registration techniques adopt static resampling techniques that are specific to certain feature geometry. Typical non-adaptive interpolation methods such as nearest neighbor, bilinear, and cubic resampling yield decreasing degrees of high-frequency information fidelity. However high frequency aliasing artifacts present in imageries need to be preserved for detection of sub-pixel features. In general, higher is the order of interpolation, the smoother is the resampled image and lesser is the local contrast information. Our investigations revealed that resampling of under sampled imagery requires information about the content of the scene being imaged to implement an adaptive method for preservation of sub pixel features. Cellular Automata based modeling has been found to be effective in achieving an intelligent hybridization of the existing interpolation strategies. Feature specific information is used to construct an adaptive resampler that adjusts itself with the image features to maintain sub pixel detection capabilities as well as accurate interpolation of large scale structures.

In this paper we investigate the possibility of using cellular neural network along with adaptive kernel strategy for improving the feature matching as well as resampling accuracies of automatic image registration. Intelligent feature modelling, effective context representation and adaptive resampling are the salient features of this work. Accuracy of developed methodologies has been compared with contemporary approaches using satellite images of Bhopal and Chandrapur cities in India.

\section{Experiment}

\subsection{Dataset description}

Different satellite images of Bhopal and Chandrapur have been used as test images for comparing the performance of various algorithms. The details of the satellite data used for these investigations are summarized in (Table 1). 120 test points have been selected for the accuracy estimation and these points are uniformly distributed over the study area. Transformation functions have been formulated independent of the test points. The ground truthing information has been collected over Bhopal and Chandrapur during October \& November 2012 respectively. Differential Global Positioning System (DGPS) has been used for survey to ensure a centimeter level accuracy. 
Table 1. Details of dataset

\begin{tabular}{|c|c|c|c|c|}
\hline $\begin{array}{c}\text { S. } \\
\text { No }\end{array}$ & Image & Satellite & $\begin{array}{c}\text { Date of } \\
\text { procurement }\end{array}$ & $\begin{array}{c}\text { Resolution } \\
(\mathrm{m})\end{array}$ \\
\hline 1 & LISS 4 & IRS-P6 & November, 2012 & 5.8 \\
\hline 2 & LISS3 & IRS-P6 & November, 2012 & 23.5 \\
\hline 4 & PAN & CARTOSAT-1 & November, 2012 & 2.5 \\
\hline
\end{tabular}

\subsection{Methodology}

Edges of master (reference image) and slave (image to be registered) images are detected using Canny operator and the boundary information along with the pixel values are used to obtain an initial object representation (details in sec 1.2.1). The images are then clustered using mixed density kernel based Support Vector Random Field (SVRF) Clustered features are further optimized using corset approach to reduce the complexity of shape modeling (details in sec 1.2.1). These object representations are modeled and interpreted using Cellular Neural Network (CNN) for effective feature matching (details in sec 1.2.2). Scale Invariant Feature Transform (SIFT) vectors of both images are refined using object information and are matched to devise the transformation function (details in sec 1.2.3). Slave image is transformed using the transformation function and is resampled with adaptive resampling strategy (details in sec 1.2.4). A schematic representation of adopted methodology is presented in (Fig. 1).

\subsubsection{Object extraction}

Once the edges of slave and master images are detected using Canny operator, Cellular Automata based region growing strategy is adopted to have approximate extraction of objects. Each pixel is assigned a state, namely "B" for boundary pixel, "NB" for non-boundary pixel, and "NR" for non-region pixels. The "NB" pixel state is changed to "NR" iteratively if it is near to a boundary pixel. The whole procedure is repeated until no further state change is experienced, thereby detecting different objects in the image.

Further clustering is accomplished using Support Vector Random Field along with mixture density kernel. Feature specific information derived using CA is utilised for determination of clustering parameters (seed pixel position, number of clusters) rather than adopting a random approach. In mixture density kernel appraoches, kernels are formulated automatically based on ensembles, and is adopted as it avoids the static nature of usual kernel tricks. Spectral and spatial information are incorporated to the kernels using a composite kernel strategy that utilises Cellular Automata rules. Preferably a weighted combination of kernels are

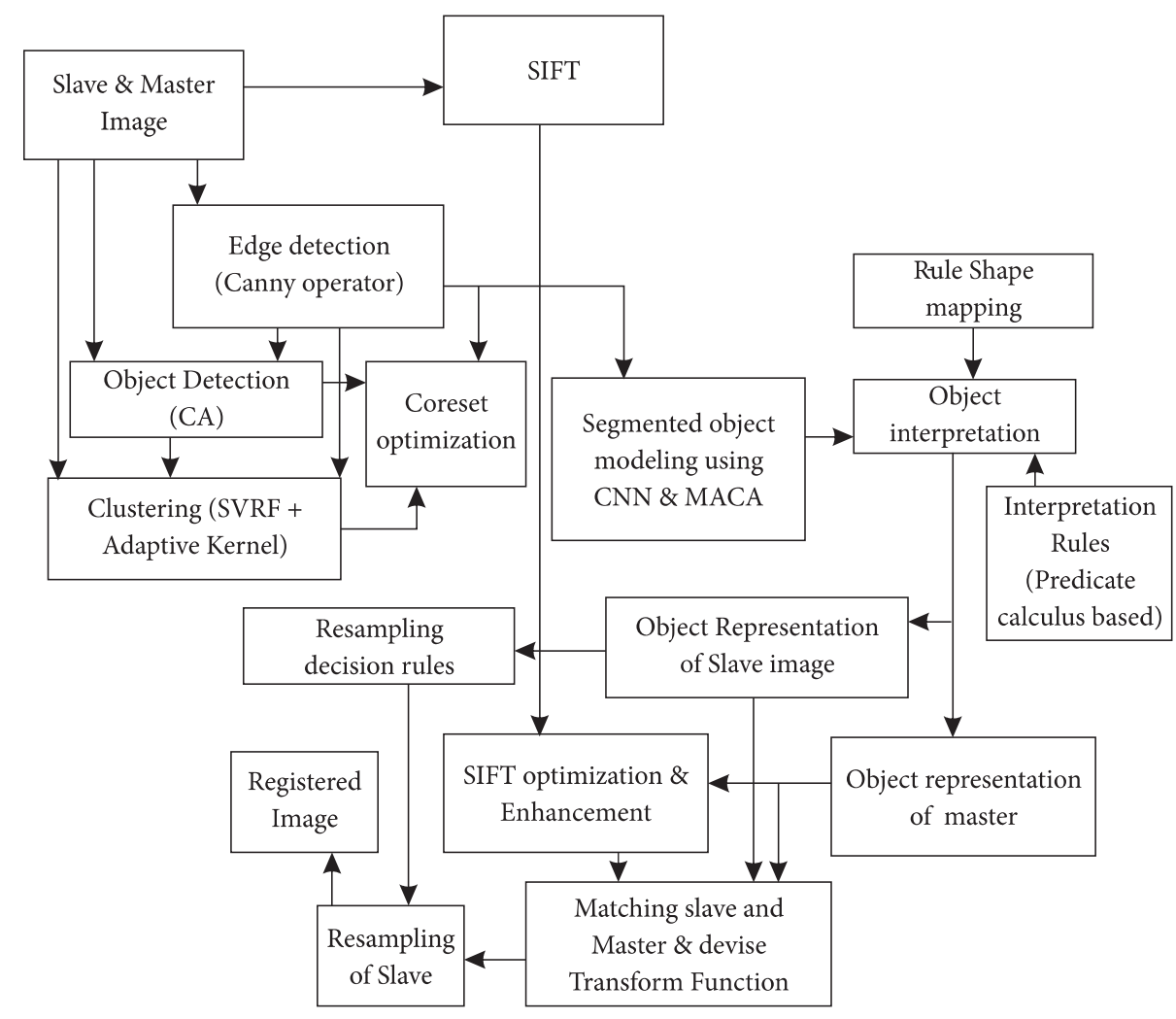

Fig. 1. Methodology of work flow 
adopted as discussed in (Hosseini, Homayouni 2009) such that $K\left(P, P_{i}\right)=\mu K_{x}\left(P, P_{i}\right)+(1-\mu) K_{y}\left(P, P_{i}\right)$. The value of tuning parameter $\mu$ is adjusted based on feature characteristics.

Clustered objects are further optimized using corset based approach since a reduction in number of pixels is required to accomplish object modeling in acceptable complexity range. Core set based approximations help to map features to lower class CA configurations without losing original shape. The parameters required for coreset generation are derived from edge information as well as abstract object representations.

\subsubsection{Feature matching}

Corresponding features in both images are detected by intelligently interpreting them. $\mathrm{CNN}$ along with GA can be effectively used to find rules that iterates from a given initial state to a desired final state. This inverse mapping or evolution is exploited to model feature shapes in both images, and $\mathrm{CNN}$ rules used to evolve a particular feature is used to distinguish it. Rules corresponding to various features are thus deducted and are stored in a prolog DB. In addition to feature interpretation, these rules are also used to guide mutation and crossover of GA to increase efficiency. The inverse evolution can be attained in lesser than $n \log (n)$ time, provided that the features will converge to lower class CA configurations.

\subsubsection{Transformation}

Once the corresponding features are modelled; master and slave images are aligned in accordance with the corresponding features. SIFT feature vectors of both images are refined using object information for computing the corresponding points. Once corresponding points in both images are determined; transformation function is computed to determine the correspondence between the remaining points of slave and master images. Different transformation functions are available over literature and prominent approaches include Thin Plate Spline (TPS), Multi Quadtratic (MQ), Weighted mean (WM) and Piecewise Linear (PL). We have adopted a weighted mean approach as suggested by Zagorchev and Goshtasby (2006) because WM is preferred over TPS, MQ, and PL when a very large set of control points with positional inaccuracies is given. WM is advantageous as it uses an averaging process that smoothes the noise and does not require the solution of a very large system of equations.

\subsubsection{Adaptive resampling}

Finally slave image intensities need to be interpolated over the transformed frame work and an adaptive approach is proposed to preserve the high frequency aliasing artifacts. Different interpolation techniques such as Nearest Neighbour (NN), Bi Linear (BL), Kaiser Damped (KD) 16, Bi-cubic (CC) and Spline (Camann et al. 2010; Wyawahare 2009; Australian Geoscience portal 2012) are combined at different scales based on specific image features. Feature information along with image scale is used to select the appropriate resampler. Images are transformed to Laplacian pyramid representation and are further categorized depending on features using resampling rules stored in Prolog Data Base). NN interpolation is adopted for small and random features in first level in the pyramid, while $\mathrm{BL}$, cubic spline, or KD-16 interpolation is used for subsequent levels. Pyramid transformation is then inverted to obtain the registered image. An important consequence of this approach is that accuracy will be enhanced at various situations, especially when an object is smaller than a single pixel but exhibits high local contrast. This is because our approach gives similar results to nearest neighbor at very fine scales, but with a cubic spline interpolant's structure superimposed. This structure comes from levels farther down in the Laplacian pyramid, where the local contrast from the immediate neighborhood at every scale is effectively combined with the sub-pixel feature.

\section{Results}

Investigations of registration process over various satellite images revealed that considerable success has been achieved with the approach. Different recent image registration techniques have been compared with the proposed method and the accuracy has been verified on diverse image datasets. Normalized Cross Correlation Coefficient (NCCC) and Root Mean Square (RMSE) values along with execution time have been compared to evaluate the efficiency of traditional approaches with reference to proposed technique. NCCC measures the similarity between images and ranges from [0-1] with a value of unity indicating perfectly registered images. RMSE value indicates the error in registration and a least value is preferred for a perfect registration. These values have been calculated in meters with reference to 120 independent test points. The execution time of different approaches have been also analyzed and is categorized as high ( $>1 \mathrm{~min}$ ), medium ( $30-60 \mathrm{sec})$, and low ( $<30 \mathrm{sec})$. Ground truthing 
have been done by means of Differential Global Positioning System (DGPS) survey over the study areas using Trimble R3 DGPS equipment. Visual results of the method are given in (Fig. 1) and results of observations are as summarized in (Table 2).

Results from the table indicates that $\mathrm{CNN}$ based approach is performing better when compared to its traditional approaches, since for all image sets (LISS4, LISS 3 and LANSAT) Normalized Cross Correlation Coefficient (NCCC) is more and Root Mean Square Error (RMSE) is less in case of the proposed method. The visual results of few features extracted by system are given below, which also reveals the accuracy of $\mathrm{CNN}$ approach.

Detected SIFT feature points and their corresponding horizontal and vertical mapping are shown in (Fig. 2). These figures illustrate the capability of system to effectively optimize the feature points using the contextual rules. Results of registration of a LISS4 image with Cartosat image is given in (Fig. 3). The intelligent interpretation of features have been utilized to
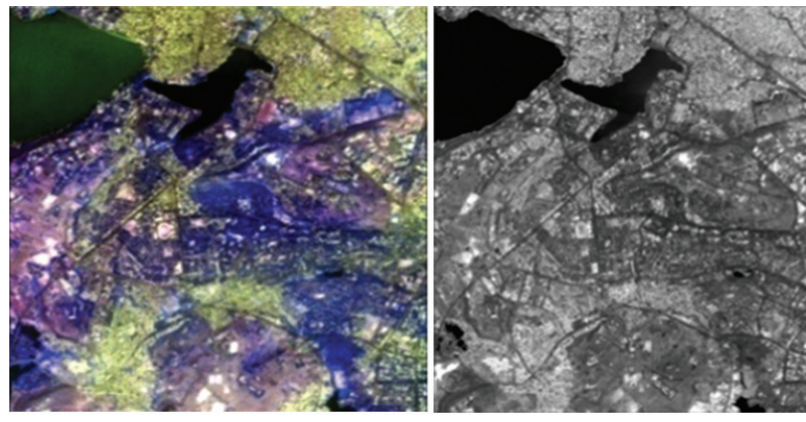

a) Slave image

b) Master image
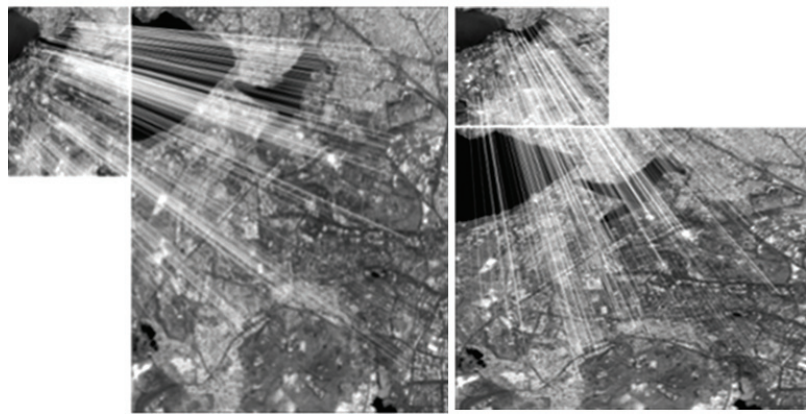

c) Horizontal Alignment

d) Vertical Alignment

Fig. 2. Registration result

Table 2. Accuracy comparison

\begin{tabular}{|c|c|c|c|c|c|c|c|c|}
\hline \multirow{2}{*}{$\begin{array}{l}\text { S. } \\
\text { No }\end{array}$} & \multicolumn{2}{|c|}{ TEST DATA } & \multirow{2}{*}{ TECHNIQUE } & \multirow{2}{*}{ NCCC } & \multirow{2}{*}{$\begin{array}{c}\mathrm{RMSE} \\
(\mathrm{m})\end{array}$} & \multirow{2}{*}{$\begin{array}{l}\text { EXE- } \\
\text { CUTION } \\
\text { TIME }\end{array}$} & \multirow{2}{*}{ PREFERENCE } & \multirow{2}{*}{ REMARKS } \\
\hline & $\begin{array}{l}\text { Master } \\
\text { Image }\end{array}$ & $\begin{array}{l}\text { Slave } \\
\text { Image }\end{array}$ & & & & & & \\
\hline 1 & Cartosat & LISS4 & $\begin{array}{c}\text { Curve based } \\
\text { (Peng et al. 2007) }\end{array}$ & 0.59 & 4.82 & low & $\begin{array}{c}\text { Presence of linear } \\
\text { features }\end{array}$ & $\begin{array}{c}\text { Enhanced by contour } \\
\text { information }\end{array}$ \\
\hline 2 & Cartosat & LISS4 & $\begin{array}{c}\text { Surface } \\
\text { (Silva et al. 2005) }\end{array}$ & 0.61 & 3.85 & low & $\begin{array}{l}\text { Availability } \\
\text { of contour } \\
\text { information }\end{array}$ & $\begin{array}{l}\text { Depends on } \\
\text { interpolation } \\
\text { methodology }\end{array}$ \\
\hline 3 & Cartosat & LISS4 & $\begin{array}{l}\text { Moment based } \\
\text { (Wu et al. 2004) }\end{array}$ & 0.53 & 3.63 & low & $\begin{array}{c}\text { Presence of rigid } \\
\text { features }\end{array}$ & $\begin{array}{c}\text { Matches features of } \\
\text { same shape }\end{array}$ \\
\hline 4 & Cartosat & LISS4 & $\begin{array}{l}\text { Morphology based } \\
\text { (Plaza et al. 2007) }\end{array}$ & 0.78 & 0.76 & High & - & $\begin{array}{l}\text { Enhanced by using } \\
\text { SIFT features }\end{array}$ \\
\hline 5 & Cartosat & LISS4 & $\begin{array}{l}\text { Mutual Information } \\
\text { (MI) Based } \\
\text { (Karvir et al. 2008) }\end{array}$ & 0.67 & 1.57 & low & $\begin{array}{c}\text { Intensity variations } \\
\text { are captured and } \\
\text { matched }\end{array}$ & $\begin{array}{l}\text { Enhance by feature } \\
\text { based approaches }\end{array}$ \\
\hline 6 & Cartosat & LISS4 & $\begin{array}{c}\text { Fourier } \\
(\mathrm{Xu}, \text { Varshney 2009) }\end{array}$ & 0.77 & 1.25 & High & $\begin{array}{l}\text { Noisy images } \\
\text { with gray level } \\
\text { variations at } \\
\text { boundaries }\end{array}$ & $\begin{array}{l}\text { Enhanced by assigning } \\
\text { variable weights to IFT }\end{array}$ \\
\hline 7 & Cartosat & LISS4 & $\begin{array}{c}\text { Wavelet } \\
\text { (Maino, Foresti 2011) }\end{array}$ & 0.85 & 0.95 & High & $\begin{array}{c}\text { Enhanced by } \\
\text { Contourlet based } \\
\text { method, SIFT } \\
\text { feature matching }\end{array}$ & $\begin{array}{l}\text { Enhanced by time and } \\
\text { frequency domain }\end{array}$ \\
\hline 8 & Cartosat & LISS4 & $\begin{array}{c}\text { Neural Network based } \\
\text { (Wang et al. 2008) }\end{array}$ & 0.63 & 2.5 & High & $\begin{array}{c}\text { Prior terrain } \\
\text { knowledge }\end{array}$ & - \\
\hline 9 & Cartosat & LISS4 & $\begin{array}{c}\text { Fuzzy based } \\
\text { (Chung et al. 2009) }\end{array}$ & 0.58 & 1.94 & High & $\begin{array}{c}\text { Presence of linear } \\
\text { features }\end{array}$ & $\begin{array}{c}\text { Combined neural } \\
\text { approach }\end{array}$ \\
\hline 10 & Cartosat & LISS4 & $\begin{array}{c}\text { SIFT based } \\
\text { (El Rube et al. 2009) }\end{array}$ & 0.87 & 1.48 & High & - & $\begin{array}{l}\text { Enhanced by object } \\
\text { specific matching }\end{array}$ \\
\hline 11 & Cartosat & LISS4 & Proposed Method & 0.53 & 0.98 & Low & $\begin{array}{l}\text { Resolution effect } \\
\text { \& noise effect are } \\
\text { nullified }\end{array}$ & $\begin{array}{c}\text { Enhanced by entropy } \\
\text { concepts }\end{array}$ \\
\hline
\end{tabular}




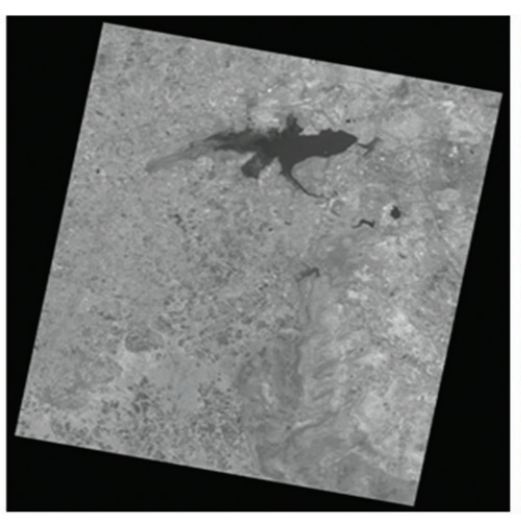

a) Master image



b) Slave image

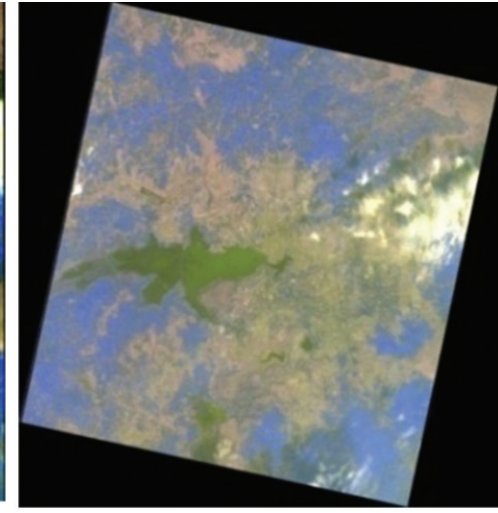

c) Registered image

Fig. 3. Registration results

effectively align the images and to adaptively resample without much loss of sub pixel information.

The main disadvantage of the method is its computational complexity which can be made good by corset optimization and similar approximation techniques. Complexity can be further reduced by storing the detected rule variations, and optimization methods such as genetic algorithm can be exploited to optimize the strategy. This research provides a basic framework and further investigations are needed to optimize it. Integration of fuzzy approach to the inverse resolution also seems to be promising as fuzzy / neutrosophic cognitive maps can be exploited for effectively organizing and selecting the Cellular $\mathrm{Au}$ tomata rule sets.

\section{Conclusions}

Feature shape modeling and context knowledge representation are two important factors in distinguishing features, and lack of its consideration has hindered the accuracy of traditional feature based registration techniques. We discussed a CNN based approach that could effectively model feature shapes and contextual knowledge for accurate registration. We have illustrated the integration of these techniques to different aspects of image registration, namely feature matching and resampling. Recent random modeling and intelligent methodologies have been adopted to present a framework for the purpose. The integration of proposed framework to SIFT feature based approach for effective enhancement has been also investigated. Investigations have revealed that the method outperforms contemporary approaches in terms of accuracy and complexity. We have also suggested a new resampling method that increases the accuracy of interpolation in the regions of the images which often have sub-pixel fidelity in under sampled data.

Paper provides a framework for CA based feature shape modeling. Complexity of the approach has been considerably reduced using corset and SIFT based approximation. Proposed system has proved to be intelligent with reference to accurate registration and resampling. Disambiguation of features, enhanced detection, self learning, minimal human interpretation, and reliability are features of the system. Further investigations are needed on the improvement of proposed framework, especially on parallelizing and optimizing different operations for complexity reduction. Effective representation of different context rules also needs further improvement and techniques such as fuzzy cognitive maps seem to be promising in this context. While the proposed method shows promising results in our early experiments, there is considerable work to be done in precisely characterizing the situations in which it performs optimally.

\section{Acknowledgement}

This research work has been carried out at Maulana Azad National Institute of Technology-Bhopal, India. Authors wish to acknowledge Dr Appukuttan K. K. along with other faculties of the institute for their helpful discussions and support towards this research work.

\section{References}

Agarwal, P. K.; Aronov, B.; Sharir, M. 2001. Exact and approximation algorithms for minimum-width cylindrical shells, Discrete Computational Geometry 26(3): 307-320. http://dx.doi.org/10.1007/s00454-001-0039-6

Australian Geoscience portal [online], [cited 22 January 2012]. Available from Internet: http://www.ga.gov.au/ 
Camann, K.; Thomas, A.; Ellis, J. 2010. Resampling considerations for registering remotely sensed images, in Proceedings of the IEEE Southeast Conference (SoutheastCon), 18-21 March, 2010, Charlotte-Concord, NC, 159-162.

Chow, C. K.; Tsui, H. T.; Lee, T. 2004. Surface registration using a dynamic genetic algorithm, Journal of Pattern Recognition 37(1): 105-117.

http://dx.doi.org/10.1016/S0031-3203(03)00222-X

Chung, F; Deng, Z.; Wang, S. 2009. An adaptive fuzzy-inference-rule-based flexible model for automatic elastic image registration, IEEE Transactions on Fuzzy Systems 17(5): 995-1010. http://dx.doi.org/10.1109/TFUZZ.2009.2020154

Cvejic, N.; Canagarajah, C. N.; Bull, D. R. 2006. Image fusion metric based on mutual information and Tsallis entropy, Electronic Letters 42(11): 626-627. http://dx.doi.org/10.1049/el:20060693

El Rube, I. A.; Sharks, M. A.; Salem, A. R. 2009. Image registration based on multi-scale SIFT for remote sensing images, in 3 rd International Conference on Signal Processing and Communication Systems, 28-30 September, 2009, Omaha, NE, $1-5$.

Fonseca, L. M. G.; Costa, M. H. M. 1997. Automatic registration of satellite images, in Proceedings of $10^{\text {th }}$ Brazilian symposium of Computer Graphic and Image Processing (SIBGRAPI), 12-16 May, 1997, IEEE Computer Society, Los Alamitos, California, 219-226.

Gouveia, A. R.; Metz, C. T.; Freire, L.; Klein, S. 2012. 3D-2D image registration by nonlinear regression, in Proceedings of 9th IEEE International Symposium on Biomedical Imaging (ISBI), 2-5 May, 2012, Brazil, 1343-1346.

http://dx.doi.org/10.1049/el:20060693

Hong, G.; Zhang, Y. 2008. Wavelet based technique for image registration techniques for high resolution satellite imagery, Computers and Geosciences 34(2): 1708-1720.

http://dx.doi.org/10.1016/j.cageo.2008.03.005

Hosseini, R. S.; Homayouni, S. 2009. A SVMS-based hyper spectral data classification algorithm in a similarity space, in Workshop on Hyper spectral Image and Signal Processing: Evolution in Remote Sensing - WHISPERS Ф09, 25 June, 2009, Grenoble, France, 1-4.

Jack, J. J.; Roux, C. 1995. Registration of 3-D images by genetic optimization, Pattern Recognition Letters 16: 823-841. http://dx.doi.org/10.1016/0167-8655(95)00051-H

Jankó, Z.; Chetverikov, D.; Ekárt, A. 2006. Using a genetic algorithm to register an uncelebrated image pair to a 3D surface model, Engineering Applications of Artificial Intelligence 19(1): 269-276. http://dx.doi.org/10.1016/j.engappai.2005.10.002

Jian, B.; Vemuri, B. C. 2005. A robust algorithm for point set registration using mixture of Gaussians, in Proceedings of 10th IEEE International Conference on Computer Vision (ICCV 2005), 17-20 October, 2005, IEEE Computer Society, Beijing, China, 1246-1251.

Karvir, H. V.; Skipper, J. A.; Repperger, D. W. 2008. Entropy selective mutual information-based image registration, in Aerospace and Electronics IEEE National Conference (NAECON), 16-18 July, 2008, Fairborn, Ohio, 173-178.

Klein, S; Staring, M.; Pluim, J. P. 2007. Evaluation of optimization methods for non-rigid medical image registration using mutual information and B-Splines, IEEE Transactions on Image Processing 16(12): 2879-2890.

http://dx.doi.org/10.1109/TIP.2007.909412
Lee, C. H.; Schmidt, M.; Murtha, A.; Bistritz, A.; Sander, J.; Greiner, R. 2005. Support vector random fields for spatial classification, in Proceedings of 9th European Conference on Principles and Practice of Knowledge Discovery in Databases (PKDD), 3-7 October, 2005, Porto, Portugal. 196 p.

Liu, J. Wu, H. 2012. A new image registration method based on frame and gray information, in Proceedings of 2012 International Conference on Computer Distributed Control and Intelligent Enviromental Monitoring, CDCIEM-2012, 5-6 March, 2012, Beijing, China, 48-51.

Lowe, D. G. 2004. Distinctive image features from scale-invariant key points, International Journal of Computer Vision 60(2): 91-110. http://dx.doi.org/10.1023/B:VISI.0000029664.99615.94

Maino, G.; Foresti, G. L. 2011. A probabilistic framework for complex wavelet based image registration, in 16th International Conference on Image Analysis and Processing - ICIAP 2011, 14-16 September, 2011, Ravenna, Italy. Part I. Berlin, Heidelberg: Springer-Verlag, 9-18.

Malviya, A.; Bhirud, S. G. 2009. Wavelet based image registration using mutual information, in Proceedings of International Conference on Emerging Trends in Electronic and Photonic Devices \& Systems, ELECTRO '09, IEEE computer society, 3-5 August, 2009, Mumbai, India, 241-244.

Mercier, G.; Lennon, M. 2003. Support vector machines for hyperspectral image classification with spectral-based kernels, IEEETransactions of Geoscience and Remote Sensing 45(3): 123-130.

Mitchell, M.; Crutch, J. P.; Das, R. 1996. Evolving Cellular Automata with genetic algorithms: a review of recent work, in Proceedings of First International Conference on Evolutionary Computation and Its Applications (EvCAX96), 20-22 May, 1996 Moscow, Russia, 120-130.

Mohanalin, J.; Kalra, P. K.; Kumar, N. 2009. Mutual information based rigid medical image registration using normalized tsallis entropy and type II fuzzy index, Journal of Medical Image Processing 43(2): 112-118.

Orovas, C.; Austin, J. 1997. A cellular system for pattern recognition using associative neural networks, in Proceedings of IEEE International Workshop on Cellular Neural Networks and their Application, 14-17 April, 1997, 2(4): 665-669.

Peng, W; Tong, R.; Qian, G.; Dong, J. 2007. A new curvesbased local image registration method, in 1st International Conference on Bioinformatics and Biomedical Engineering (ICBBE 2007), 6-8 July, 2007, Wuhan University, China, 984-988.

Plaza, A.; Le Moigne, J.; Netanyahu, N. S. Morphological feature extraction for automatic registration of multispectral images, in IEEE International Geoscience and Remote Sensing Symposium (IGARSS 2007), 23-28 July, 2007, Barcelona, Spain, 421-424.

Porway, J.; Wang, K.; Yao, B.; Zhu, S.-C. 2008. A hierarchical and contextual model for aerial image understanding, in Proceedings of IEEE Computer Society Conference on Computer Vision and Pattern Recognition (CVPR 2008), 24-28 June, 2008, Anchorage, Alaska, USA, 49(7): 1-8.

Schnitzspan, P.; Fritz, M.; Schiele, B. 2008. Hierarchical support vector random fields: joint training to combine local and global features, in Proceeding of $10^{\text {th }}$ European Conference on Computer Vision, Marseille-France, Palais des Congrès Parc Chanot, 12-18 October, 2008, 53(5): 527-540.

Sikdar, B.; Paul, K.; Biswas, G. P.; Yang, C.; Bopanna, V.; Mukherjee, S.; Chaudhuri, P. P. 2000. Theory and application of GF(2P) 
Cellular Automata as on-chip test pattern generator, in Proceedings of 13th International Conference on VLSI Design, 4-5 January, 2000, Mumbai, India, 556-561.

Silva, L.; Bellon, O. R. P.; Boyer, K. L. 2005. Precision range image registration using a robust surface interpenetration measure and enhanced genetic algorithms, Pattern Analysis and Machine Intelligence: IEEE Transactions 27(5): 762-776. http://dx.doi.org/10.1109/TPAMI.2005.108

Srivastava, A. N. 2004. Mixture density Mercer kernels: a method to learn kernels directly from data, in Proceedings of $4^{\text {th }}$ SIAM International Conference on Data Mining, 22-24 April, 2004, Lake Buena Vista, Florida, USA.

Viola, P.; Wells, W. M. 1997. Alignment by maximization of mutual information, International Journal of Computer Vision 24(2): 147-154. http://dx.doi.org/10.1023/A:1007958904918

Wang, S; Sheng, Lei; Chang, F. 2008. Image registration based on neural network, in International Conference on Information Technology and Applications in Biomedicine (ITAB 2008), 30-31 May, 2008, Corfu, Greece, 74-77.

Wu, J.; Xie, J. 2004. Zernike moment-based image registration scheme utilizing feedforward neural networks, in Fifth World Congress on Intelligent Control and Automation (WCICA 2004), 15-19 June, 2004, Hangzhou, China, 4046-4048.
Wyawahare, M. V.; Patil, P. M.; Abhyankar, H. K. 2009. Image registration techniques: an overview, International Journal of Signal Processing, Image Processing and Pattern Recognition 2(3): 11-28.

Xu, M.; Varshney, P. K. 2009. A subspace method for fourierbased image registration, IEEE, Geoscience and Remote Sensing Letters 6(3): 491-494.

http://dx.doi.org/10.1109/LGRS.2009.2018705

Zagorchev, L.; Goshtasby, A. 2006. A comparative study of transformation functions for nonrigid image registration, IEEE Transactions on Image Processing 15(3): 529-538. http://dx.doi.org/10.1109/TIP.2005.863114

Zitová, B.; Flusser, J. 2003. Image registration methods: a survey, Image Vision Computation 21(11): 977-1000. http://dx.doi.org/10.1016/S0262-8856(03)00137-9

Pattathal Vijayakumar ARUN. He has completed his Masters from NIT-Bhopal, and is currently pursuing $\mathrm{PhD}$. His main area of interest are artificial Intelligence, spatial mining, and image processing. 